\title{
A Constraint Guided Progressive Sequential Mining Waterfall Model for CRM
}

\author{
Bhawna Mallick ${ }^{1}$, Deepak Garg ${ }^{1}$ and P. S. Grover ${ }^{2}$ \\ ${ }^{1}$ Department of Computer Science \& Engineering, Thapar University, Patiala, India \\ 2 Department of Computer Science \& Engineering, GTBIT, Delhi, India
}

\begin{abstract}
CRM has been realized as a core for the growth of any enterprise. This requires both the customer satisfaction and fulfillment of customer requirement, which can only be achieved by analyzing consumer behaviors. The data mining has become an effective tool since often the organizations have large databases of information on customers. However, the traditional data mining techniques have no relevant mechanism to provide guidance for business understanding, model selection and dynamic changes made in the databases. This article helps in understanding and maintaining the requirement of continuous data mining process for CRM in dynamic environment. A novel integrative model, Constraint Guided Progressive Sequential Mining Waterfall (CGPSMW) for knowledge discovery process is proposed. The key performance factors that include management of marketing, sales, knowledge, technology among others those are required for the successful implementation of CRM. We have studied how the sequential pattern mining performed on progressive databases instead of static databases in conjunction with these CRM performance indicators can result in highly efficient and effective useful patterns. This would further help in classification of customers which any enterprise should focus on to achieve its growth and benefit. An organization has limited number of resources that it can only use for valuable customers to reap the fruits of CRM. The different steps of the proposed CGP-SMW model give a detailed elaboration how to keep focus on these customers in dynamic scenarios.
\end{abstract}

Keywords: customer relationship management, key performance indicators, data mining techniques, constraints, sequential patterns, progressive databases, incremental mining

\section{Introduction}

In the competitive market today, it has become essential to maintain regular customers along with the effort made to acquire new ones. This necessitates developing long-term and pleasant relationships with regular customers. The main objective of any customer relationship management application is to understand and satisfy the requirements of customers. Data mining brings various techniques together to discover rules and construct models from databases. It is the process in which the enterprise extracts its useful knowledge from a lot of incomplete and random information generated through various processes of the enterprise. This information also includes the customer purchasing behavior, a must for the customer relationship management. In the past, researchers generally applied traditional data mining methods like statistical surveys to study customer behavior. However, now the need for advanced techniques of data mining like sequential pattern extraction is well realized. These techniques search through a database to obtain implicit, previously unknown and potentially useful information. It is a must for an enterprise to provide high quality products and services, but it also must react appropriately to changes in customer needs. Data mining techniques can be applied to identify useful regular customer behavior patterns from large amounts of customers' transaction data for better decision making. However, most of them are used to generate predictions and describe behaviors. Relatively little research has focused on mining changes in the databases collected over time. Moreover, the customer behavior has been studied often from the customer demographic perspective. There are customer behavioral variables, like monetary, specific time duration that can be used to differentiate customer 
contributions to a business. These aspects need to be incorporated as constraints while mining to achieve business specific information regarding the CRM.

This study attempts to integrate the constraints identified from the customer value analysis, along with the sequential pattern mining on progressive database. So, it establishes a method of mining customer behavior rules in the dynamic environment. In this study, we have proposed the Constraint Guided Progressive Sequential Mining Waterfall (CGP-SMW) model that can assist managers in developing better marketing strategies and planning for successful implementation of CRM. The contribution of this research article can be summarized as below:

1. To understand the need of customer value analysis for CRM.

2. To study the importance of sequential pattern mining over other data mining technique for analyzing consumer behavior.

3 . To signify the role of incorporating constraints in mining process referred to as constraint-based sequential pattern mining (CBSPM).

4. To focus on applying CBSPM on progressive databases instead of static databases. This would aid in getting useful knowledge under dynamic environment.

5. To identify the key performance parameters for CRM. These factors could be used to decide the frequency for application of mining technique on progressive databases.

6. To establish a Constraint Guided Progressive Sequential Mining Waterfall (CGP-SMW) model for CRM that can be used by business managers for decision-making.

The rest of this article is organized as follows: In Section 2, we briefly cover the association of customer relationship management (CRM) and the data mining. In Section 3, the related work based on different data mining techniques used for CRM is cited. The constraint based sequential pattern mining on progressive databases is induced in Section 4. Integration of CRM key performance indicators along with the mining process is discussed in Section 5 to have dynamic useful results. Based on these concepts, we have proposed CGP-SMW model for successful implementation of CRM, using constraint based sequential pattern mining in Section 5. We have concluded our study in Section 6.

\section{Customer Relationship Management and Data Mining}

Customer Relationship Management (CRM) is being used by any organization to identify the problems of customers and to improve the consistency with them. CRM is the utilization of customer related information or knowledge to deliver relevant products or services to customers (Parvatiyar et al., 2001; Collins, K. 2001). The main objective of CRM is to retain high value customers and to acquire new customers by providing high satisfaction. This further requires meeting customer needs, improving customer service and performing customer analysis. It also helps in maximizing the corporate profit to a measurable extent. Customer satisfaction provides results in the form of increased purchased volumes, repetitive purchases by regular and new customers, generation of new business in the form of references and word of mouth. However, due to the industrial automation, most enterprises have generated a mass of business data. Often the useful and valuable information is hidden in the enterprise database which is required for customer analysis.

To achieve the benefits of CRM, many enterprises are using data mining techniques. Data mining is a decision support process that allows finding valuable information from a huge raw data in the database. It helps decision-makers in getting useful regular patterns and forecasting trends for repeating purchases by regular or new customers. It provides useful information in an easy to understand form for decision-making. The main feature of data mining involves extraction, transformation, analysis, and formulation of models for the masses of commercial data in the customer databases. Different data mining methods are available to extract the information and analyze the problem of any organization to attract more customers for buying their products. Statistical analysis, probability theory, regression analysis, factor analysis, discriminant 
analysis and cluster analysis are some of them. In recent times, methods like decision tree, artificial neural networks and rules induction have become quite popular.

Rule induction is an area of machine learning in which formal rules are extracted from a set of observations. The rules extracted may represent a full scientific model of the data, or merely local patterns in the data. These rules could be formulated based on association mining or sequential pattern mining. Sequential pattern mining algorithms address the problem of discovering the existent frequent sequences in a given database. Sequential pattern mining is different from association rule mining, where the events are linked by time. Sequential patterns are generated using the correlation between transactions while association rule mining gives patterns based on intra transaction relationships. Sequential pattern is a sequence of item sets that frequently occur in a specific order; all items in the same item set are supposed to have the same transaction time value or a value within a time gap. A sequential pattern is a sequence having support greater than or equal to a minimum threshold, called the minimum support. The support of a sequence is the percentage of data sequences containing the sequence. The support calculation for association rules mining is transaction-based. However, sequential pattern mining makes use of sequencebased support calculation. It is obvious from this discussion that the sequential pattern mining is more useful than any other data mining technique for CRM. It can play a vital role in studying the customer purchasing behavior due to its sequential tendencies to formulate strategies and marketing plans.

\section{Related Work}

Many previous CRM-related researches used different data mining techniques to analyze and understand customer behavior and characteristics. For example, Kim, Jung, Suh, and Hwang (2006) adopted decision tree to classify the customers and further develop CRM strategies. This study is based on customer segmentation in accordance with their lifetime value so as to facilitate CRM. Hwang, Jung, and Suh (2004) used logistic regression to segment customers. They used customer loyalty as the basis of segmentation. Kim and Street (2004) proposed a system which makes use of neural networks and genetic algorithm for customer targeting. Kim (2006) used logistic regression and neural networks for feature selection to predict churn. However, Baesens (2004) identified the slope of the customer lifecycle based on Bayesian network classifier. Sinha and Zhao (2008) adopted decision tree and logistic regression for churn prediction by incorporating domain knowledge into data mining. Tsai and $\mathrm{Lu}(2009)$ used hybrid neural network for churn prediction. However, customer value analysis is required when the focus is on retaining valuable customers and acquiring new customers for CRM. This further mandates studying and analyzing customer purchasing behavior. Association rule mining and sequential pattern mining can only be useful in such cases of CRM.

For different purposes in CRM, there have been researches on the utilization of association rules and sequential patterns. Sequential patterns can be used to predict future complaint (Lariviere \& Van den Poel, 2005) and network banking churn (Chiang, Wang, Lee, \& Lin, 2003). Aggarwal, Procopiuc, and Yu (2002) conducted market basket analysis to identify association rules. Changchien, Lee, and Hsu (2004) used neural networks and association rules obtained from market basket analysis to develop on-line personalized sales promotion. Tsai and Chen (2010) used association rules to select variables for churn prediction. There are limited studies based on customer value analysis using sequential pattern mining in the literature. Those available have shortcoming as discussed in next section.

\section{Problem Formulation}

The research studies based on sequential pattern mining may generate huge or very few patterns based on the support threshold provided. For a larger support threshold, the mining result could have almost no or very few patterns that satisfy the threshold. While in case there is a small support threshold, the mining would result in generating enormous patterns. Incorporation of additional constraints into mining is 
a possible and effective solution to this problem. There is much importance of the mechanism of constraint-based mining as it allows the user to have focused results in form of application semantics and business specific patterns. Besides allowing user exploration and control, the paradigm allows many of these constraints to be pushed deep inside mining. This prunes the search space of patterns to those of interest to the user and achieves superior performance. The mining process which incorporates user-specified constraints to reduce search space and derive only the user-interested patterns is called constraint-based sequential pattern mining (CBSPM). There has been some research work in the area of CBSPM. Hosseini, Maleki, and Gholamian (2010) used RFM values with K-means algorithm to classify the customer loyalty. Cheng and Chen (2009) used RFM values with K-means and rough set theory to segment customers. Chen, $\mathrm{Kuo}, \mathrm{Wu}$, and Tang (2009) identified purchasing patterns in the form of sequential patterns. Huang, Chang, and $\mathrm{Wu}$ (2009) adopted K-mean and clustering to analyze the characteristics of customers based on RFM values. Chiang (2010) mined association rules of customer values. Mallick et al (2012) used CFM with the mining algorithms to give constraint based sequential patterns.

However, a big question arises how to decide for the constraints that should be incorporated within the mining process? These studies have identified the constraints based on particular ap-

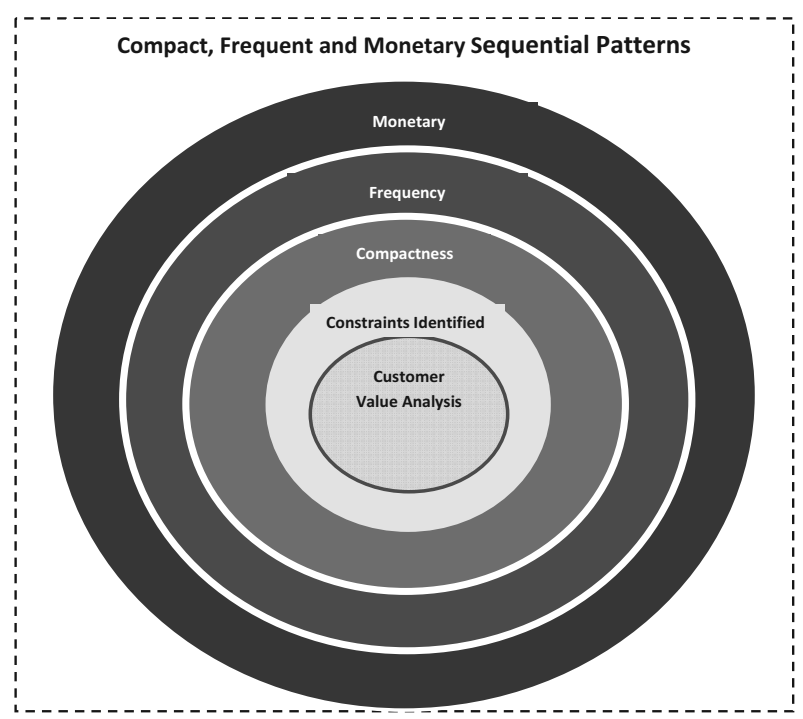

Figure 1. Identification of constraints based on Customer Value Analysis (core) of CRM. plication. The relevance of constraints from CRM perspective is not well studied. This question is addressed in our previous work, Mallick et al (2013). It is well justified in this study that customer value analysis for CRM can be vital for the selection and incorporation of constraints in the mining of the customers' sequential databases. In our study, it has been proved that the use of compactness, frequency and monetary constraints can help to acquire new customers, retain regular customers and preserve valuable customers. Once the constraints are identified, we can use them within the mining process itself to get the constraintbased sequential patterns (CBSP) [as shown in Figure 1]. These discovered patterns can further be given scores or priority to classify the customers based on purchasing patterns.

However, this method works in a one-time fashion, that is, mine the entire database and obtain the set of results. It handles mining on static databases, the one that does not change with time. Needless to state for CRM, databases are not static and are usually appended with new data sequences, conducted by either existing or new customers. The appending might invalidate some existing patterns whose supports become insufficient with respect to the currently updated database, or might create some new patterns due to the increased supports. It is obvious that the customer shopping transaction database grows daily due to the appending of newly purchased items by existing customers. It can also be due to the insertion of new shopping sequences for new customers. The constraint based sequential pattern mining method is not suitable for handling this situation because the result mined from the old database is no longer valid on the updated database. Moreover, it is inefficient to mine the updated databases from scratch.

Transaction appending, deletions and modifications are the possible updates that lead to sequence databases grow incrementally. As databases evolve, the problem of maintaining sequential patterns over a significantly long period of time becomes essential. The information from the old frequent sequences, i.e., the support of frequent sequences can be re-used to minimize the computational cost. With respect to the same support threshold, the incremental mining aims to find out the new set of sequential patterns after database updating without re-mining the whole customer purchasing 
database. But, the problem to have the current state of the database persists with the incremental constraint based sequential pattern mining. This is due to the presence of previous sequential patterns that would become irrelevant and new sequential patterns might appear. When sequential patterns are generated, the newly arriving patterns may not be identified as frequent sequential patterns. This is because of the existence of old data and sequences. Further to the problem, the results may depict the obsolete sequential patterns that are not frequent recently. The incremental constraint based mining does not consider deletion of the obsolete data from the sequence database. This may cause lack of interest among the CRM users. It is noted that CRM users are usually more interested in the recent data than in the old ones.
The solution comes in form of progressive sequential pattern mining which deals with a progressive database. This type of mining not only adds new data to the original database, but also removes obsolete data from the database. The sequential pattern mining with a static database finds sequential patterns in the database that do not change over time. The sequential pattern mining with an incremental database corresponds to the mining process where there are new data arriving with time. As for the sequential pattern mining with a progressive database, new data are added into the database and obsolete data are removed simultaneously [detail study is done in Figure 2]. Therefore, one can find the most up-to-date sequential patterns that are not influenced by obsolete data. This has been well justified in our study made on progressive databases, Mallick et al. (2013).

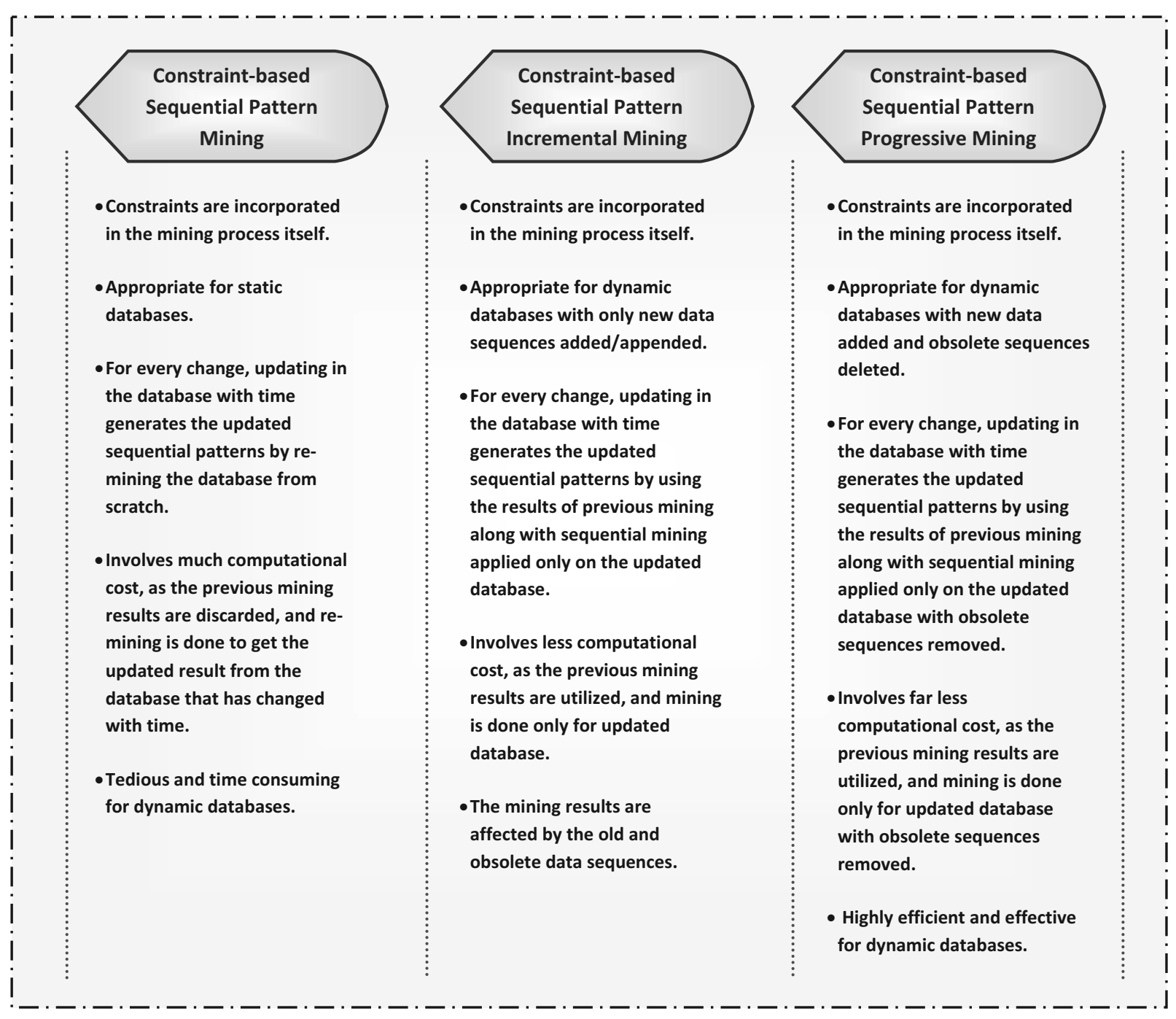

Figure 2. Characteristics of constraint-based sequential mining on normal, incremental and progressive databases. 


\section{Proposed Work}

With this discussion, the significance of CBSPM on progressive databases for CRM has been realized. There are other important aspects, as mentioned below, that requires to be studied in detail:

1. Who are the CRM users to make use of customers' purchasing progressive sequence databases?

2. What are the different ways in which CRM users access the customers' purchasing progressive sequence databases?

3. How is it decided on the frequency of access to the customers' purchasing progressive sequence databases?

The answers to all these queries could be made by identifying the factors that contribute to the successful implementation of CRM. The type/number of CRM users will depend on the nature of CRM factors. These CRM features will also cause difference in the access methods and the frequency of usage of the customers' purchasing progressive sequence databases. In the next section, we have identified the key performance contributors for CRM.

\subsection{CRM Key Performance Indicators}

Different factors contribute to the implementation of best CRM. These include management of different aspects, that is, technology, sales, marketing, customer service, supply chain, inventory control, knowledge and retail. Technology can help to reach market and publicize the products to the customers. It could be used to make convenient, easier and better services to them like making use of online payment, maintaining customer call centers for feedbacks and complaints etc. The sales management is often the backbone of CRM as it is responsible for enhancing the sales of the products. To achieve it, it could make use of efficient planning and strategy formulation. The other important factor is marketing management which ensures the appropriate suggestions for new products. It takes care of the strategy based on customers, company and competitor analysis. Every effort goes in vain if we are not able to maintain the customer once acquired. This requires good customer support and service. It includes answering the customer's query, satisfying the customers for their complaints and assessing their valuable suggestions for future improvements and advancements.

The very first level of customer satisfaction is achieved by delivering the required product on time. This could only be maintained by appropriate inventory control and supply chain management. Appropriate inventory control ensures timely delivery of new and fresh products without the need of much storage requirement. However, the supply chain management requires proper coordination of all departments of organization. The storage of raw materials, production and delivery to customers that is from point-of-origin to point-of-consumption needs to be well managed. Knowledge management is another crucial factor of CRM, as it helps to make forecast of products based on previous data. It also enables to attract the customers for the products and ideas of the enterprise. The employees of the organization can improve their future by making benefit for the same. Lastly, retail management contributes for the CRM by delivering the manufactured products to the customers through short and convenient channels. Due to the importance and relevance of these factors to the success of the CRM, we have termed them as the Key Performance Indicators (KPIs) for the CRM in this article.

Figure 3 depicts the identified eight key performance indicators along with their desired and minimum priorities for CRM implementation of any enterprise on a scale from 1 to 10. The desired priority level specifies a specific KPI to achieve in normal scenarios for the successful execution of CRM. However, under any circumstances, the KPI should at least maintain the minimum priority level for the CRM.

CRM through constraint based sequential mining is more efficient in acquiring new customers, increasing value of existing customers and retaining good customers. But this mining has to be performed on progressive database with the frequency and access mechanism governed by the CRM key performance indicators. 


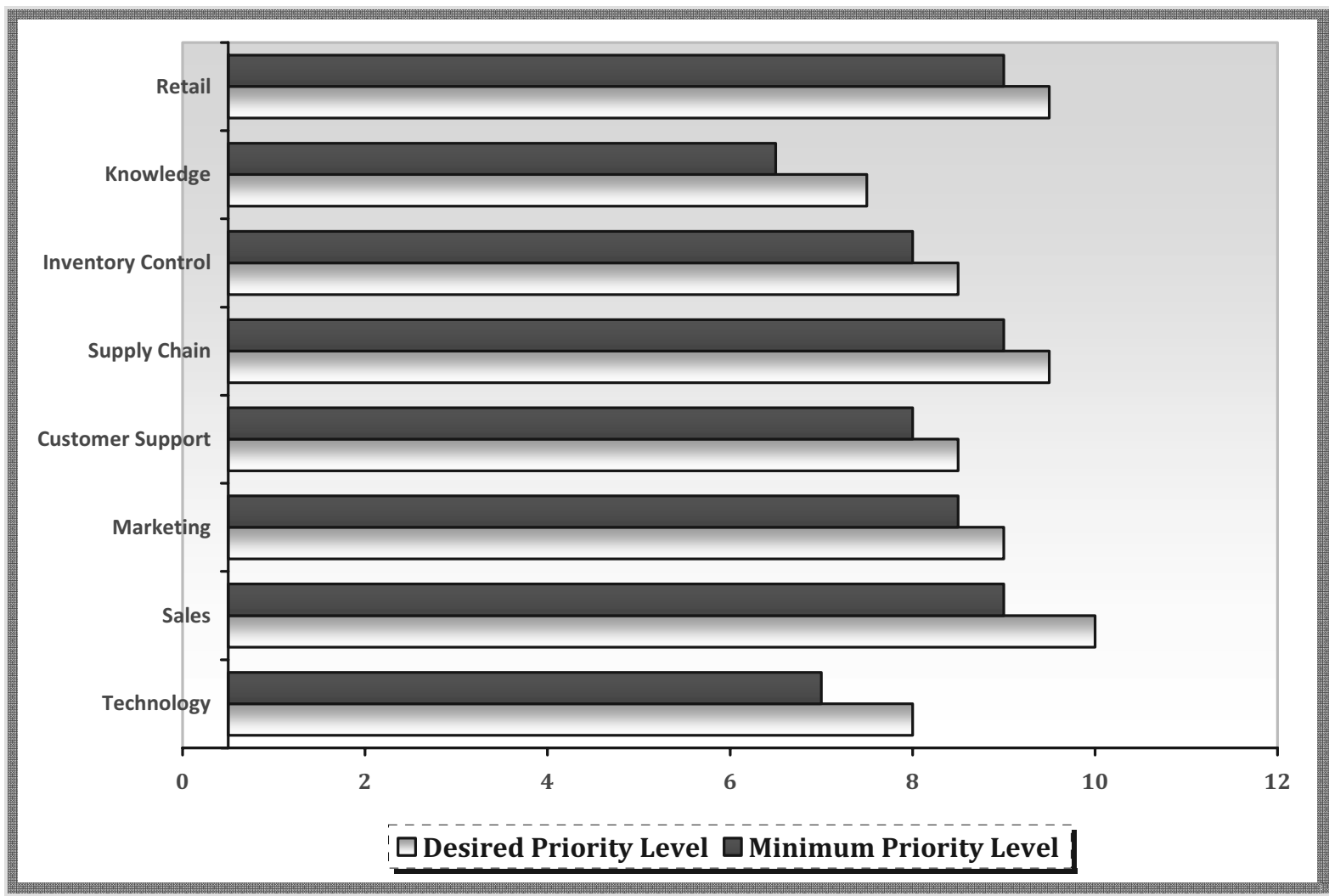

Figure 3. CRM key performance indicators along with the desired and minimum priority level for any enterprise.

\subsection{Constrained Guided Progressive Sequential Mining Waterfall (CGP-SMW) Model}

It has already been mentioned in Section 3 and Section 4 that incorporation of constraints in the mining process itself could give useful and more relevant business- centric patterns. There has to be a basis for the selection of constraints and for the CRM application the customer value analysis is recommended. The identified constraints should be further used to generate sequential patterns by using them within the mining process. This will improve the value of the results obtained. The discovered patterns could be further used to classify the customers based on the scores or priority assigned to each resultant sequential pattern. These customers are the ones on which any enterprise should focus and concentrate their limited resources to achieve the goals of the CRM. However, looking at the dynamic and versatile nature of the CRM due to customer purchasing behavior, the discovered patterns lose their significance with time. So it becomes necessary to maintain the progressive databases, instead of static or incremental. This will generate the most relevant and useful patterns giving valuable customers for CRM. This leads to an obvious query how to maintain the progressive database, that is, are we required to change and modify the database with every addition, modification or deletion? If we are to follow this, then the progressive database management would become tedious, expensive and time consuming process itself. To take this into account, the frequency of maintaining the progressive database could be decided based on the CRM's key performance indicators. The factors which are significant for the successful implementation of the CRM have been identified. Based on the requirements of these KPIs the progressive database could be maintained.

To get valuable customers for any enterprise for the objectives of CRM relevant in both scalable and dynamic environments, the steps as given below, can be followed:

1. Perform the customer value analysis. 
2. Identify the constraints relevant for the business based on step 1 .

3. Conduct the sequential mining by incorporating the constraints in step 2 .

4. Classify the customers based on the patterns generated in step 3.

5. Identify KPIs for CRM and use them to decide the frequency of maintaining progressive database.

6. Perform sequential mining on progressive databases to get the valuable customers with the changing environment.

As prominent, these steps need to be performed in a specific sequence, that is, each step needs to be executed after the successful completion of the previous one. At the same time, the results of earlier step are being used by the next step for its implementation. It is the typical behavior of the waterfall model. We can, therefore, consider our model as a Constraint Guided Progressive - Sequential Mining Waterfall (CGPSMW) model, as given in Figure 4.

This model is iterative in nature to take care of the changes with time. The parameters of cus- tomer value analysis could differ in dynamic environment. For example, we have suggested acquiring new customers, maintaining regular customers and preserving valuable customers as important parameters for customer value analysis. These could be modified, added or appended with other parameters by any enterprise in competitive market. It would require further analysis and this would lead to identification of different constraints altogether. For example, for our suggested parameters compactness, frequency and monetary constraints are identified. However, if the enterprise intends to change the parameter of customer value analysis based on the number of purchases made by the customer in single transaction, then the length constraint has to be incorporated in the mining process. As the sequential pattern mining is based on constraints, it is to be performed again with every alteration in step 1, leading to change in step 2 and all the following steps, as the model inherits waterfall nature. In fact, it is not necessary that we need to always transform from step 1 to take care of scalability in modern business scenario. For example, if required to change the CRM's KPI to modify the frequency of maintenance

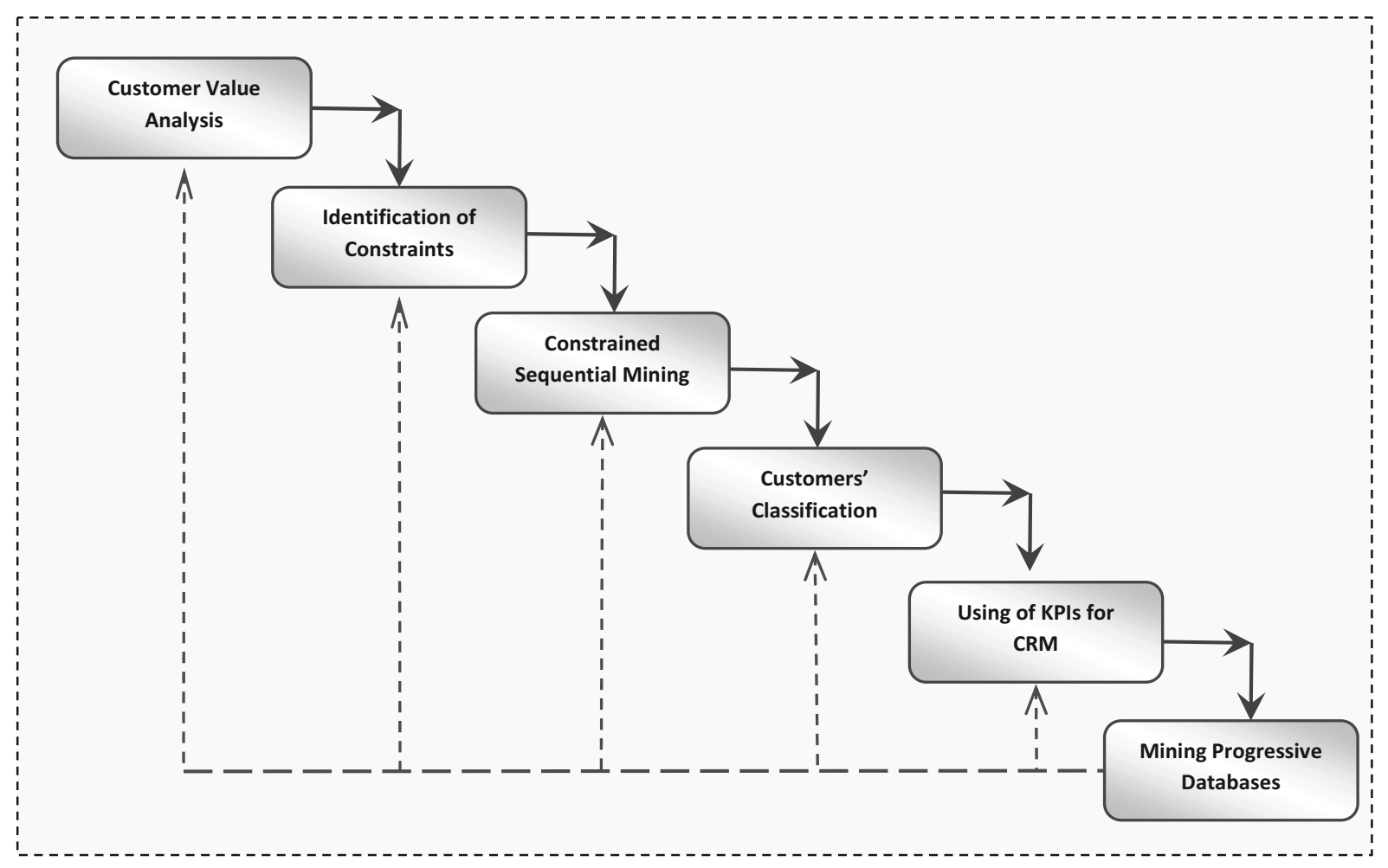

Figure 4. Constraint guided progressive-sequential mining waterfall (CGP-SMW) model. 
of progressive databases, then the iteration is limited to steps 5 and 6.

We can conclude that the CGP-SMW model is hence iterative waterfall model as shown in Figure 4. The detailed and elaborative version of this model is given in Figure 5.

\section{Conclusions}

Customer relationship management is essential effectively to compete today's market. The profitability of an enterprise depends on the

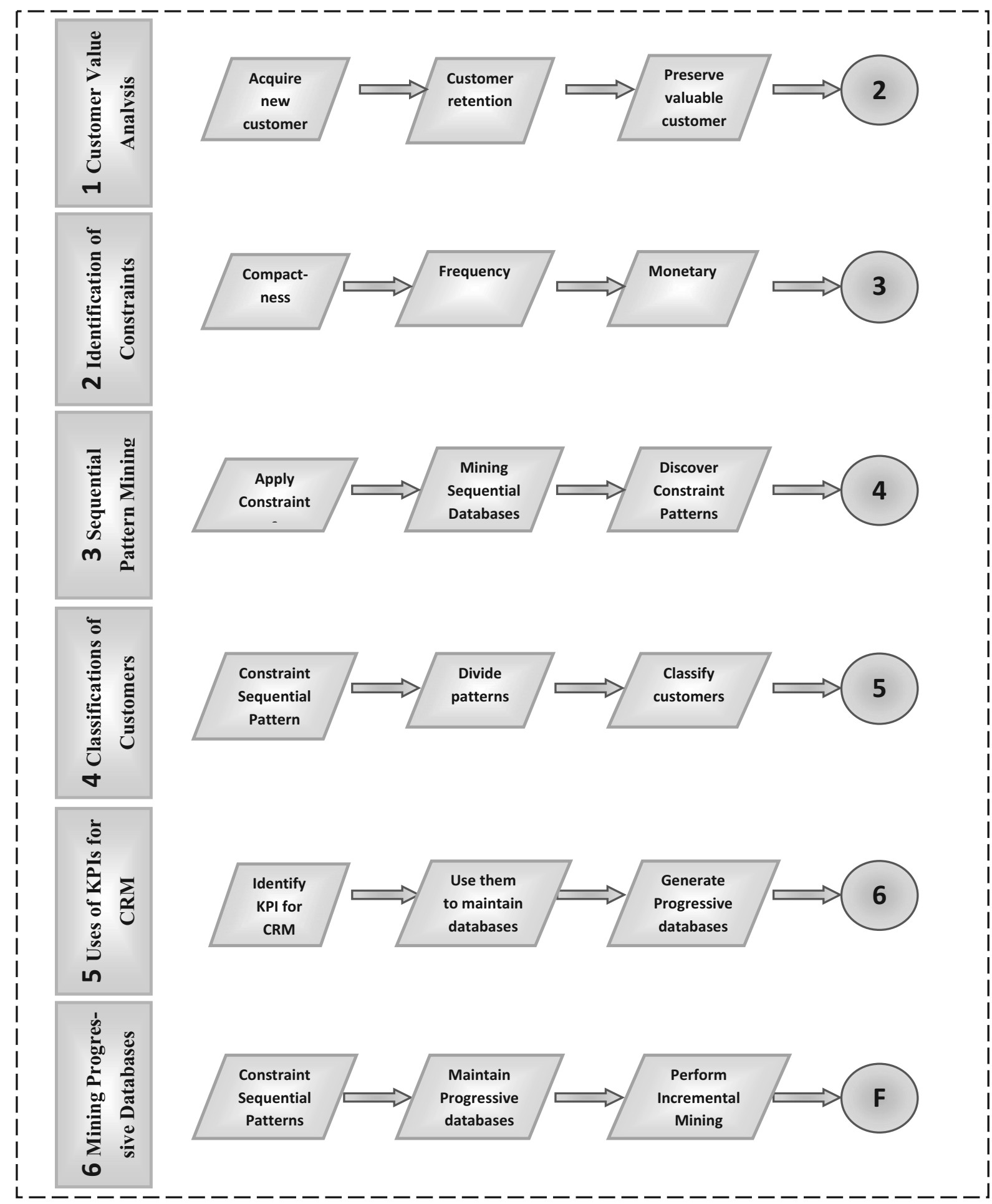

Figure 5. The detailed version with each step elaborated for the proposed CGP-SMW model. 
use of information about customers and meeting their needs effectively. CRM based on CBSPM can help enterprises obtain and maintain competitive advantage. CRM based on CBSPM can understand customer's needs, improve customer's satisfaction, gain more share in the market for the enterprise and ultimately promote its profitability. The constraints could be identified based on customer value analysis and applied on progressive databases to take care of the scalable market. Studies reveal that almost $70 \%$ of the efforts put in the CRM initiative goes down the drain and the CRM fails due to inability of the performing companies to resolve certain key issues (D. K. Rigby and D. Ledingham, 2004). So the performance factors for CRM like sales, marketing, technology etc. should be utilized to decide on the access mechanism and frequency of using the progressive database. The proposed model, CGP-SMW, if used appropriately, will help in getting the information about the customers any enterprise should focus on for achieving the objectives of CRM.

\section{Acknowledgements}

The authors would like to thank the Editor and anonymous referees for providing valuable comments and constructive suggestions. We express our gratitude to the Galgotias College of Engineering and Technology, Greater Noida for the support provided to complete this research work.

\section{References}

[1] C. C. Aggarwal, C. Procopiuc, P. S. Yu, Finding localized associations in market basket data. IEEE Transactions on Knowledge and Data Engineering, 14 (2002), 51-62.

[2] B. Baesens, G. Verstraeten, D. Vanden Poel, M. EGMONT-Petersen, P. VAN Kenhove, J. VANTHIENEN, Bayesian network classifiers for identifying the slope of the customer lifecycle of long-life customers. European Journal of Operational Research, 156 (2004), 508-523.

[3] S. Changchien, C. F. LeE, Y. J. Hsu, On-line personalized sales promotion in electronic commerce. Expert Systems with Applications, 27 (2004), 3552.
[4] Y. L. Chen, M. H. Kuo, S. Y. Wu, K. TAnG, Discovering recency, frequency, and monetary (RFM) sequential patterns from customers' purchasing data. Electronic Commerce Research and Applications, $\mathbf{8}$ (2009), 241-251.

[5] C. H. Cheng, Y. S. Chen, Classifying the segmentation of customer value via RFM model and RS theory. Expert Systems with Applications, 36 (2009), 4176-4184.

[6] D. A. Chiang, Y. F. WANG, S. L. LeE, C. J. Lin, Goal-oriented sequential pattern for network banking churn analysis. Expert Systems with Applications, 25 (2003), 293-302.

[7] W. Y. CHIANG, To mine association rules of customer values via a data mining procedure with improved model: An empirical case study. Expert Systems with Applications, (2010).

[8] K. Collins, Analytical CRM: Driving Profitable Customer Relationships, Strategic Planning, SPA12-7120, (2001).

[9] D. K. Rigby, D. Ledingham, CRM Done Right. Harvard Business Review, (November 2004), pp. 118-129.

[10] S. M. S. Hosseini, A. MALEKI, M. R. GHOLAMian, Cluster analysis using data mining approach to develop CRM methodology to assess the customer loyalty. Expert Systems with Applications, 37 (2010), 5259-5264.

[11] S. C. Huang, E. C. Chang, H. H. Wu, A case study of applying data mining techniques in an outfitter's customer value analysis. Expert Systems with Applications, 36 (2009), 5909-5915.

[12] H. Hwang, T. Jung, E. SuH, An LTV model and customer segmentation based on customer value: A case study on the wireless telecommunication industry. Expert Systems with Applications, 26 (2004), $181-188$

[13] S. Y. KIM, T. S. Jung, E. H. SuH, H. S. Hwang, Customer segmentation and strategy development based on customer lifetime value: A case study. Expert Systems with Applications, 31 (2006), 101-107.

[14] Y. S. KIM, Toward a successful CRM: Variable selection, sampling, and ensemble. Decision Support Systems, 41 (2006), 542-553.

[15] Y. S. KIM, W. N. StreET, An intelligent system for customer targeting: A data mining approach. Decision Support Systems, 37 (2004), 215-228.

[16] B. LARIVIERE, D. VAN DEN POEL, Investigating the post-complaint period by means of survival analysis. Expert Systems with Applications, 29 (2005), 667-677.

[17] B. Mallick, D. Garg, P. S. Grover, CFM- PrefixSpan: A pattern growth algorithm incorporating compactness and monetary. International Journal of Innovative Computing, Information and Control, $\mathbf{8}(7(\mathrm{~A}))(2012), 4509-4520$.

[18] B. Mallick, D. Garg, P. S. Grover, CRM Customer Value based on Constrained Sequential Pattern Mining. International Journal of Computer Applications, (2013), in press. 
[19] B. Mallick, D. Garg, P. S. Grover, Progressive CFM-Miner: An algorithm to mine CFMSequential patterns from a progressive database. International Journal of Computational Intelligent Systems, 6(2) (2013), 209-222.

[20] A. Parvatiyar, J. N. Sheth, Customer relationship management: Emerging practice, process, and discipline. Journal of Economic and Social Research, 3 (2001), 1-34.

[21] A. P. SINHA, H. ZHAO, Incorporating domain knowledge into data mining classifiers: An application in indirect lending. Decision Support Systems, 46 (2008), 287-299.

[22] C. F. TSAI, M. Y. CHEN, Variable selection by association rules for customer churn prediction of multimedia on demand. Expert Systems with Applications, 37 (2010), 2006-2015.

[23] C. F. Tsai, Y. H. LU, Customer churn prediction by hybrid neural networks. Expert Systems with Applications, 36 (2009), 12547-12553.

Received: January, 2013

Revised: January, 2014

Accepted: May, 2014

Contact addresses:

Bhawna Mallick

Department of Computer Science \& Engg

Thapar University

Patiala

India

e-mail: bhawna.mallick@gmail.com

Deepak Garg

Department of Computer Science \& Engg

Thapar University

Patiala

India

P. S. Grover

Department of Computer Science \& Engg

GTBIT

Delhi

India
BHAWNA MALLICK received her B.Tech in Computer Technology from Nagpur University, India and M.Tech in Information Technology from Punjabi University, Patiala, India. She received her $\mathrm{PhD}$ degree in the area of developing efficient algorithms for incremental mining from Thapar University. She is presently working as Professor \& Head of Department of Computer Science \& Engineering at Galgotias College of Engineering \& Technology, Greater Noida affiliated to UP Technical University, India. She has more than 15 years of industry and academic experience with organizations like Infosys Technologies Ltd, Chandigarh, India and NIIT Technologies Ltd, New Delhi, India. Her research interest is data mining, focusing on sequential mining from progressive databases and she has several publications in international journals.

DEEPAK GARG received his Ph.D. in the area of efficient algorithm design from Thapar University. He is certified on latest technologies from Sun for Java Products, IBM for Web services and Brain bench for programming concepts. He is Senior Member of IEEE (Institute of Electrical and Electronics Engineers), USA, Executive Member of IEEE Delhi Section and Secretary of IEEE Computer Society, Delhi Section. He is Life Member of ISTE, CSI , IETE (Institute of Electronics and Telecommunication Engineers), ISC (Indian Science Congress), British Computer Society and ACM, UK. He started his career as a software engineer in IBM Corporation Southbury, CT, USA and then with IBM Global Services India Pvt Ltd, Bangalore, India. He is presently working as Professor at Thapar University, Patiala.Deepak has 37 publications in international journals and conferences. He is on the editorial boards of seven international journals. His active research area is data structure, algorithms and data mining.

P. S. GROVER received his Master's degree and doctorate from Delhi University, Delhi, India. He has widely travelled and delivered invited talks/keynote addresses at many national/international conferences/seminars and workshops. He is on the editorial boards of four international journals. He has written 9 books and many of his articles have appeared in several books published by IEEE of USA. He has published more than 100 research papers in international and national journals and conferences including published by IEEE, ACM and Springer. $\mathrm{He}$ is presently Director General at Guru Tegh Bahadur Institute of Technology, GGS Indraprastha University, Delhi, India. Formerly he was Dean \& Head of Computer Science Department, Delhi University, Delhi, India. Prof Grover is a member of IEEE Computer Society. His current research interests are: component based and aspect-oriented software engineering, autonomic embedded systems. 